\title{
Review Article \\ S. N. Bernstein Type Estimations in the Mean on the Curves in a Complex Plane
}

\author{
J. I. Mamedkhanov and I. B. Dadashova \\ Institute of Mathematics and Mechanics, Baku State University, \\ 370148 Baku, Azerbaijan \\ Correspondence should be addressed to I. B. Dadashova, irada-dadashova@rambler.ru \\ Received 3 June 2009; Accepted 3 August 2009 \\ Recommended by John Rassias
}

The present paper discusses in the metric $L_{p} S$. N. Bernstein type inequalities of the most general kind on very general accessible classes of curves in a complex plane. The obtained estimations, generally speaking, are not improvable.

Copyright (c) 2009 J. I. Mamedkhanov and I. B. Dadashova. This is an open access article distributed under the Creative Commons Attribution License, which permits unrestricted use, distribution, and reproduction in any medium, provided the original work is properly cited.

\section{Introduction}

The estimations connecting the norms of derivatives of polynomials with the norm of the polynomial itself are usually called the Markov-Bernstein type estimations. Therewith, the similar global estimation in the metric $C_{[-1,1]}$ was obtained by Markov. Bernstein considered the similar estimation in the metric $C_{[0,2 \pi]}$ for trigonometric polynomials and also local estimation in the metric $C_{[-1,1]}$. Bernstein type local estimation, which is precise in the sense of order in the metric $C_{[-1,1]}$, was obtained by Dzjadyk. Further, Dzjadyk considered this estimation in a complex plane. Earlier, such a global estimation in a complex plane in the metric $C$ was obtained by Mergelyan [1]. Validity of such estimations on arbitrary compacts in a complex plane in the metric $C$ was shown in the papers of Lebedev and Tamrazov [2]. Similar problems in the mean, namely, in the metric $L_{p}$ have their own specification that does not allow to consider such estimations on wide classes of sets in a complex plane. For a long time, the validity of such estimations was known on very narrow classes of curves of a complex plane. These results, in particular, are given in [3].

One of the theorems with appropriate Bernstein inequality is announced in [4]. Some auxiliary statements, by means of which such inequalities are proved, are in [4].

In the sequel, we will need the following facts. 


\section{Preliminary Notes}

Let $\Gamma$ be a closed curve in a complex plane with parametric representation $z=z(t)(0 \leq t \leq l$, $l$ is the length of $\Gamma)$ of diameter $d^{*}\left(d^{*}=\sup _{t, \tau \in \Gamma}|t-\tau|\right)$ the function $z=\psi(\omega)$ maps the exterior of a unit circle $\gamma_{0}$ onto the exterior of $\Gamma$, and $z=\psi_{0}(\omega)$ maps the interior of $\gamma_{0}$ on the interior of $\Gamma$; the functions $w=\varphi(z)$ and $w=\varphi_{0}(z)$ are inverse to the functions $z=\psi(\omega)$ and $z=\psi_{0}(\omega)$, respectively; $\Gamma_{1+\rho}$ is a level line of the curve $\Gamma$ corresponding to the equation $|\varphi(z)|=1+\rho(\rho>0)$.

Let $t$ be some fixed point on $\Gamma_{1+\rho}(\rho>0)$, let $d(t, \Gamma)=d$ be the distance from the point $t$ to the curve $\Gamma, \Gamma_{\delta}^{*}(t)=\{z \in \Gamma:|z-t|<\delta\}$, and $\theta_{t}^{*}(\delta)=\theta_{t}^{*}(\delta, \Gamma)=\operatorname{mes} \Gamma_{\delta}^{*}(t)$.

Let us consider a class of curves $\Gamma$, for which $\theta_{t}^{*}(\delta) \leq C(\Gamma) \delta$ for $\delta \geq 2 d$. We denote this class of curves by $S_{\theta}^{*}$. It is easy to show that the class $S_{\theta}^{*}$ coincides with the class $S_{\theta}$ introduced by Salayev [5].

Recall that the curve $\Gamma$ belongs to the class $S_{\theta}$, (Salayev's class) if there exists a constant $C(\Gamma) \geq 1$, such that $\theta(\delta) \leq C(\Gamma) \delta$, where $\Gamma_{\delta}(t)=\{\tau \in \Gamma:|t-\tau| \leq \delta\},(0<\delta \leq d) \theta_{t}(\delta)=$ mes $\Gamma_{\delta}(t)$ (Lebesgue measure), and $\theta(\delta)=\sup _{t \in \Gamma} \theta_{t}(\delta)$.

So, the following statement $[4]$ is true.

Statement $2.1\left(S_{\theta}=S_{\theta}^{*}\right)$. By $J_{\gamma}$ we denote a class of Jordan rectifiable curves $\Gamma$, for which the following relation [4]

$$
\tilde{d}^{\gamma-1}\left(t, \frac{1}{n}\right) \cdot \int_{\Gamma} \frac{|d z|}{|z-t|^{\gamma}} \leq C(\Gamma, \gamma)
$$

is valid ( $\tilde{d}$ is a distance from the point $t \in \Gamma_{1+1 / n}$ to the curve $\Gamma$ ) for the given $\gamma>1$ and all $t \in \Gamma_{1+1 / n}$.

The following statement [4] is also valid.

Statement 2.2 (If $1<\gamma_{1}<\gamma_{2}$, then $J_{\gamma_{1}} \subset J_{\gamma_{2}}$ ). We will say that the function $\theta_{t}(\delta, \Gamma) / \delta$ is almost increasing in $\delta$ uniformly in $t$, if there exists a constant $C(\Gamma)$ not depending on $t$ such that for any $\delta_{1}<\delta_{2}$ the following inequality $\theta_{t}\left(\delta_{1}, \Gamma\right) / \delta_{1} \geq C(\Gamma)\left(\theta_{t}\left(\delta_{2}, \Gamma\right) / \delta_{2}\right)$ is fulfilled.

Note that many known classes of rectifiable curves, in particular the curves of the class $S_{\theta}$ (Salayev's class), satisfy the condition that $\theta_{t}(\delta, \Gamma) / \delta$ is almost decreasing.

By $J_{\gamma}^{*}$ we denote a subclass of the class of curves $J_{\gamma}$, for which $\theta_{t}(\delta, \Gamma) / \delta$ almost decreases. For the classes of curves $J_{\gamma}$ and $J_{\gamma}^{*}$, the following statement is valid [4].

Statement 2.3. There hold the embeddings

$$
\begin{aligned}
& S_{\theta} \subset J_{\gamma}, \\
& J_{\gamma}^{*} \subset S_{\theta} .
\end{aligned}
$$

Now, let us consider the quantity

$$
\delta\left(z, \frac{1}{n}\right)=\left(\int_{\Gamma_{1+1 / n}} \frac{|d t|}{|z-t|^{2}}\right)^{-1}, \quad z \in \Gamma .
$$




\section{Main Results}

In particular, using the previously mentioned statement, we can prove the following theorems.

Theorem 3.1. Let $\Gamma$ be an arbitrary rectifiable Jordan curve on which for any $s \in(0, \infty)$ and any natural $j$ the following estimation is valid (signs $\preccurlyeq$ and $\asymp$ define an ordinal relation. Namely, $A \preccurlyeq B$ means $A \leq$ const $B$. And $A \asymp B$ means const $A \leq B \leq$ const $A$ ):

$$
\widetilde{\delta}^{s}\left(t, \frac{1}{n}\right) \int_{\Gamma} \frac{\delta^{j-s}(z, 1 / n)|d z|}{|z-t|^{j+1}} \preccurlyeq 1, \quad t \in \Gamma_{1+1 / n}
$$

where

$$
\tilde{\delta}\left(t, \frac{1}{n}\right)=\left(\int_{\Gamma} \frac{|d z|}{|z-t|^{2}}\right)^{-1}
$$

Then

$$
\left\|\delta^{j-s}\left(z, \frac{1}{n}\right) P_{n}^{(j)}(z)\right\|_{L_{p}(\Gamma)} \preccurlyeq\left\|\delta^{-S}\left(z, \frac{1}{n}\right) P_{n}(z)\right\|_{L_{p}(\Gamma)},
$$

where $P_{n}(z)$ is an algebraic polynomial of degree $n \in N, p \geq 1$.

We can also prove a theorem of independent character used in the proof of Theorem 3.1.

Theorem 3.2. Under the conditions of Theorem 3.1 on the curve $\Gamma$, whatever was the natural number $j$ and $s \in(-\infty, \infty)$ for the $j$ th-order derivative of the polynomial $P_{n}(z)$ of degree $\leq n$, for $p \geq 1$, the following inequality

$$
\left\|\frac{P_{n}^{(j)}(t)}{\| \widetilde{\delta}^{s-j}(t, 1 / n)}\right\|_{L_{p}\left(\Gamma_{1+1 / n}\right)} \leq C(\Gamma, p, j, s)\left\|\frac{P_{n}(z)}{\delta^{s}(z, 1 / n)}\right\|_{L_{p}(\Gamma)}
$$

is valid.

A special case of these theorems is similar theorems for concrete classes of curves, namely, for the following classes.

(a) $K$-quasiconformal mapping. The curve $\Gamma$, being an image of the circle under some $K$-quasiconformal mapping of the plane onto itself, is said to be $K$-quasiconformal curve. The class of curves will be denoted by $A_{k}$.

(b) We will say that the set $E$ with rectifiable Jordan curve $\Gamma=\partial E$ belongs to the class $B_{k}$ [3] for some $k$ (or $\Gamma \in B_{k}$ ), if $\Gamma \in S_{\theta}$ and satisfies the following conditions:

(1) $|\widetilde{z}-z| \asymp d(z, 1 / n)$, where for all $z \in \Gamma, \widetilde{z}=\widetilde{z}(1 / n)=\psi((1+(1 / n)) \varphi(z)), \underset{\sim}{z}=$ $\psi\left((1+(1 / n))^{-1} \varphi(z)\right)$;

(2) $|\tilde{t}-t| \preccurlyeq|\tilde{t}-z|^{k-1}|\tilde{z}-z|, \forall z, t \in \Gamma$. 
As Dzjadyk shows [3, page 393], the validity of the condition

$$
|\tilde{z}-z| \asymp d\left(\tilde{z}, \frac{1}{n}\right)
$$

that is equivalent to the following geometric property of domain $E$ [6] follows from conditions (1) and (2) of the class $B_{k}$. inequality

(3) We can connect any points of $z$ by the $\operatorname{arc} \gamma(z, \xi) \subset E$ whose length satisfies the

$$
\text { mes } \gamma(z, \xi) \preccurlyeq|z-\xi| \text {. }
$$

Furthermore, [6, Lemmas 1 and 2], the following conditions are valid for the set $E$ of the class $B_{k}$ :

(4) if $\xi \in \Omega=C E, \xi_{\Gamma}=\psi\left[\varphi(\xi)|\varphi(\xi)|^{-1}\right], \Gamma=\partial E$, then

$$
d(\xi, \Gamma) \stackrel{\text { def }}{=} \inf _{z \in \Gamma}|\xi-z| \asymp\left|\xi-\xi_{\Gamma}\right|
$$

(5) if $z \in \Gamma, \widetilde{z}_{R}=\psi[R \varphi(z)], R>1$, then

$$
d\left(z, \Gamma_{\mathrm{R}}\right) \stackrel{\text { def }}{=} \inf _{t \in \Gamma_{R}}|z-t| \asymp\left|\tilde{z}_{R}-z\right|
$$

Note that the $K$-quasiconformal curves [7] satisfy conditions (1)-(5) and relation (3.5). Consider some more general classes.

(c) We will say that $E \in H$ (or $\Gamma=\partial E \in H$ ), if conditions (4) and (5) are fulfilled.

(d) We will say that $E$ with a rectifiable boundary $\Gamma$ belongs to $D$ (or $\Gamma \in D$ ), if $\Gamma \in S_{0}$, and conditions (3) or its equivalent relation (3.5) is fulfilled for it.

Obviously, the class of the sets $D$, possessing pure geometric description, contains the classes of the sets $B_{k}$.

So, the following theorems are true.

Theorem 3.3. Let $\Gamma$ be an arbitrary rectifiable $K$-quasiconformal curve. Then, whatever was the natural number $j$ and the number $s \in(-\infty, \infty)$ for the $j$ th order derivative of the polynomial $P_{n}$ of power $\leq n$ for $p \geq 1$, the following inequality is valid:

$$
\left\|\tilde{d}^{j-s}\left(t, \frac{1}{n}\right) P_{n}^{(j)}(t)\right\|_{L_{p}\left(\Gamma_{1+1 / n}\right)} \leq C(\Gamma, p, j, s)\left\|d^{-s}\left(z, \frac{1}{n}\right) P_{n}(z)\right\|_{L_{p}(\Gamma)} .
$$

Theorem 3.4. Let $\Gamma$ for some natural $k$ belong to the class $B_{k}$. Then, whatever was the natural number $j$ and (under some additional condition on the curve $\Gamma$, Theorem 3.4 remains valid for any $s \geq 0$ (see Remark 5.1).) $s \in[0, k j /(k-1) p)$ for the $j$ th-order derivative of the polynomial $P_{n}$ of power $\leq n$ for $p \geq 1$, the following inequality is valid:

$$
\left\|d^{j-s}\left(z, \frac{1}{n}\right) P_{n}^{(j)}(z)\right\|_{L_{p}(\Gamma)} \leq C(\Gamma, p, j, s)\left\|d^{-s}\left(z, \frac{1}{n}\right) P_{n}(z)\right\|_{L_{p}(\Gamma)} .
$$


The special case of these theorems is announced in [8] and is cited in [9, 10] with incomplete proof.

Remark 3.5. The special case of Theorems 3.3 and 3.4 was also proved in [11] for curves consisting of infinitely many smooth arcs; each of these arcs has continuous curvature, and at the joint points $z_{j}(i=\overline{1, m})$ they form between themselves external angles $\alpha_{i} \pi$ such that $1<\alpha_{i}<2$, that is, on the curves of the class $W_{[1,2)}$.

In this paper, we give a complete proof of Theorems 3.3 and 3.4. Theorems 3.1 and 3.2 are proved by the same method Theorems 3.3 and 3.4 with the usage of Statements 2.1-2.3.

\section{Auxiliary Lemmas}

When proving Theorems 3.3 and 3.4 we'll need the following.

$\left(1^{\circ}\right)$ A nonnegative function $\rho(z)$ given on the plane $z$ will be said to be admissible if

$$
A(\rho)=\iint \rho^{2} d x d y<+\infty
$$

If $\mathrm{T}$ is a family of locally rectifiable curves on the plane, we put

$$
L_{\rho}(\mathrm{T})=\inf _{\gamma \in \mathrm{T}} \int_{\gamma} \rho|d z|
$$

(if $\rho$ is not measurable on $\gamma$, we assume that $\int_{\gamma} \rho|d z|=\infty$ ). If $\mathrm{P}$ is a class of admissible functions, then the quantity

$$
\lambda(\mathrm{T})=\sup _{\rho \in \mathrm{P}} \frac{L_{\rho}^{2}(\mathrm{~T})}{A(\rho)}
$$

is said to be external length of $\mathrm{T}$, and its inverse quantity

$$
\lambda^{-1}(\mathrm{~T}) \stackrel{\text { def }}{=} m(\mathrm{~T})
$$

a modulus of $\mathrm{T}$ is

$$
m(\mathrm{~T})=\lambda^{-1}(\mathrm{~T})=\inf _{\rho \in \mathrm{P}} \frac{A(\rho)}{L_{\rho}^{2}(\mathrm{~T})} .
$$

Let $\Omega$ be an arbitrary one-connected domain of a complex domain containing the point $z=\infty$; let $\bar{B}$ be a complement to $\Omega$; let $\Gamma=\partial \Omega=\partial \bar{B}$ be their common boundary; let $w=\varphi(z)$ 
be a function that conformally and univalently maps $\Omega$ onto $\Omega^{\prime}$ exterior of a unit circle and is normed by the following condition:

$$
\varphi(\infty)=\infty, \lim _{z \rightarrow \infty} \frac{\varphi(z)}{z}>0 ; \quad z=\psi(w)=\varphi^{-1}(w)
$$

$\Gamma_{1+\sigma} \stackrel{\text { def }}{=}\{t:|\varphi(t)|=1+\sigma \geq 1\}$ be a level line of the continuum $\bar{B}$; let $d(z, \sigma) \stackrel{\text { def }}{=}$ $\inf _{t \in \Gamma_{1+\sigma}}|z-t|$, for $z \in \Gamma$; let $\tilde{d}(t, \sigma) \stackrel{\text { def }}{=} \inf _{z \in \Gamma}|z-t|$, for $t \in \Gamma_{1+\sigma}$.

The following statements are valid.

Lemma $A$ (see [12, Lemma 1.2]). Let $\bar{B}$ be an arbitrary continuum with connected complement $\Omega, z_{0}=\Gamma=\partial \bar{B}, z_{1}, z_{2} \in \Omega$.

$$
\begin{array}{r}
\text { If }\left|z_{1}-z_{0}\right|>\left|z_{2}-z_{0}\right|,\left|\varphi\left(z_{1}\right)-\varphi\left(z_{2}\right)\right| \leq C_{1}\left|\varphi\left(z_{2}\right)-\varphi\left(z_{0}\right)\right| \text {, then } \\
\frac{1}{2 \pi} \ln \frac{\left|z_{1}-z_{0}\right|}{\left|z_{2}-z_{0}\right|} \leq m(\mathrm{~T})=m\left(\mathrm{~T}^{\prime}\right) \leq C\left(C_{1}, \Gamma\right),
\end{array}
$$

where $\mathrm{T}$ is a family of curves isolating the points $z_{1}$ and $z_{*}$ in $\Omega$ (in simplest cases $z_{*}=z_{0}$ ) from the points $z_{2}$ and $\infty$ and $\mathrm{T}^{\prime}=\varphi(\mathrm{T})$.

Lemma $B$ (see [13, Theorem 1$]$ ). Let $\bar{B}$ be an arbitrary continuum with connected complement. Then for $w \in \Omega^{\prime}$

$$
\left|\psi^{\prime}(w)\right| \asymp \frac{d(\psi(w), \bar{B})}{|w|-1},
$$

or

$$
\left|\varphi^{\prime}(w)\right| \asymp \frac{|\varphi(z)|-1}{d(z, \bar{B})}, \quad z=\psi(w),
$$

where $d(z, \bar{B})$ is a distance from the point $z=\psi(w)$ to $\bar{B}$.

Lemma $C$ (see [14, Lemma 1]). Let $w=F(z)$ realizes $K$-quasiconformal mapping of plane onto itself, $F(\infty)=\infty . C_{z}, C_{w}$ are, respectively, $z$ - and $w$-complex planes; $z_{j} \in C_{z}, \quad F\left(z_{j}\right)=$ $w_{j} \in C_{w},(j=1,2,3)$.

Then we have the following:

(1) the conditions $\left|z_{1}-z_{2}\right| \preccurlyeq\left|z_{1}-z_{3}\right|$ and $\left|w_{1}-w_{2}\right| \preccurlyeq\left|w_{1}-w_{3}\right|$ are equivalent, and consequently the conditions

$$
\left|z_{1}-z_{2}\right| \asymp\left|z_{1}-z_{3}\right|, \quad\left|w_{1}-w_{2}\right| \asymp\left|w_{1}-w_{3}\right|
$$

are also equivalent; 
(2) if $\left|z_{1}-z_{2}\right| \preccurlyeq\left|z_{1}-z_{3}\right|$, then

$$
\frac{\left|w_{1}-w_{3}\right|^{A}}{\left|w_{1}-w_{2}\right|} \preccurlyeq \frac{\left|z_{1}-z_{3}\right|}{\left|z_{1}-z_{2}\right|} \preccurlyeq \frac{\left|w_{1}-w_{3}\right|^{B}}{\left|w_{1}-w_{2}\right|}
$$

where $A=K^{-1}, B=K$.

Lemma $D$ (see [11]). Let $G$ be a domain with a rectifiable boundary $\Gamma$, and $\Omega=C G \quad(\infty \in \Omega$ ). If $f \in E_{p}(\Omega)(p \geq 1)$, then for any $R>1$ and for all $\rho \in(1, R]$ the following inequality holds:

$$
\|f\|_{L_{p}\left(\Gamma_{\rho}\right)} \leq R^{2 / p}\|f\|_{L_{p}(\Gamma)} .
$$

$\left(2^{\circ}\right)$ Let $\xi$ be some arbitrary fixed point lying outside of $\Gamma$, and let $d=d(\xi, \Gamma)$ be a distance from the point $\xi$ to $\Gamma, \Gamma_{\delta}(\xi)=\{z \in \Gamma:|z-\xi|<\delta\}$, and $\theta_{\xi}^{*}(\delta)=\theta_{\xi}^{*}(\delta, \Gamma)=\operatorname{mes} \Gamma_{\delta}(\xi)$.

To prove these theorems we will need the following lemmas.

Lemma 4.1. Let a rectifiable curve $\Gamma \in H$, then for a polynomial $P_{n}$ of power $\leq n$ for $p \geq 1$ the following inequality is valid for $s \in(-\infty, \infty)$ :

$$
\left\|\tilde{d}^{-s}\left(t, \frac{1}{n}\right) P_{n}(t)\right\|_{L_{p}\left(\Gamma_{1+1 / n}\right)} \leq C(\Gamma, p, s)\left\|d^{-s}\left(z, \frac{1}{n}\right) P_{n}(z)\right\|_{L_{p}(\Gamma)} .
$$

Lemma 4.2. Under conditions of Lemma 4.1 on the curve $\Gamma$, for a polynomial $P_{n}$ of power $\leq n$ for $p \geq 1, s \in(-\infty, \infty)$ and $\rho \leq 1 / n$ the following inequality is valid:

$$
\left\|d^{-S}\left(\xi, \Gamma_{R}\right) P_{n}(\xi)\right\|_{L_{p}\left(\Gamma_{1+\rho}\right)} \leq C(\Gamma, p, s)\left\|d^{-S}\left(z, \frac{1}{n}\right) P_{n}(z)\right\|_{L_{p}(\Gamma)}
$$

where under $d^{-S}\left(\xi, \Gamma_{R}\right), \xi \in \Gamma_{1+\rho}$ one understands a distance from the point $\xi, \xi=\psi(\tau)$ to the level line $\Gamma_{R}$, where $R=|\tau|(1+1 / n), \tau=\varphi(\xi)$.

Lemma 4.3. Let $\Gamma \in B_{k}$. Then whatever was a natural number $j$ and $s \in[0, k j /(k-1))$, the inequality

$$
\widetilde{d}^{s}\left(t, \frac{1}{n}\right) \int_{\Gamma} \frac{d^{j-s}(z, 1 / n)|d z|}{|z-t|^{j+1}} \leq C(\Gamma, s, j), \quad \forall t \in \Gamma_{1+1 / n}
$$

is valid.

Lemma 4.4 (see [9]). Let $\Gamma \in S_{\theta}$. Then for $\gamma>1$ and all $t \in \Gamma_{1+1 / n}$ the relation (2.1) is valid; that is, the imbedding $S_{\theta} \subset J_{\gamma}(\gamma>1)$ is valid. 
Lemma 4.5 (see [9]). Let $\Gamma \in D$. Then for $\gamma>1$ and all $z \in \Gamma$ the inequality

$$
d^{\gamma-1}\left(\xi, \frac{1}{n}\right) \int_{\Gamma_{1+1 / n}} \frac{|d t|}{|t-z|^{\gamma}} \leq C(\Gamma, \gamma)
$$

is valid.

Proof of Lemma 4.1. Let an arbitrary rectifiable curve $\Gamma \in H$. At first we consider the case $s \geq 0$. Introduce some auxiliary function

$$
S(z)=\frac{\left[\varphi^{\prime}(\tilde{z})\right]^{s} P_{n}(z)}{[\varphi(z)]^{n}}
$$

where $\tilde{z}=\widetilde{z}(1 / n) \stackrel{\text { def }}{=} \psi((1+1 / n) \varphi(z))$.

Obviously, $S(z) \rightarrow 0$, as $z \rightarrow \infty$ and each of its branchs is holomorphic in $C \bar{G}(\Gamma=$ $\partial G)$ and continuous in $\overline{C \bar{G}}$. Therefore $S \in E_{p}(C G)$. Consequently, we can apply to $S(z)$ Lemma D where by estimation of Lemma B we will have

$$
\left\|\frac{P_{n}(t)}{\tilde{d}^{s}(\tilde{t}, \Gamma) \varphi^{n}(t)}\right\|_{L_{p}\left(\Gamma_{1+1 / n}\right)} \leq C(P)\left\|\frac{P_{n}(z)}{\tilde{d}^{s}(\tilde{z}, 1 / n) \varphi^{n}(z)}\right\|_{L_{p}(\Gamma)} .
$$

Now, if we consider that $|\varphi(t)|^{n} \asymp 1$, for $t \in \Gamma_{1+1 / n}$ and the relations $d(z, 1 / n) \asymp|\tilde{z}-z| \asymp$ $\tilde{d}(\widetilde{z}, 1 / n)$, which is valid for any $\Gamma \in H$, then for the proof of (4.13) it suffices to prove the validity of the relation

$$
d(\tilde{t}, \Gamma) \asymp \tilde{d}\left(t, \frac{1}{n}\right)
$$

where $t \in \Gamma_{1+1 / n}$.

Let $t \in \Gamma_{1+1 / n}, \underset{\sim}{t} \stackrel{\text { def }}{=} \psi\left((1+1 / n)^{-1} \varphi(t)\right)$. Obviously $\underset{\sim}{t} \in \Gamma$. By the property of curves of the class $H$, we have

$$
\begin{gathered}
\tilde{d}\left(t, \frac{1}{n}\right) \asymp|t-\underset{\sim}{t}| \asymp d\left(\underset{\sim}{t}, \frac{1}{n}\right), \\
d(\tilde{t}, \Gamma) \asymp|\tilde{t}-\underset{\sim}{t}| \asymp d\left(\underset{\sim}{t}, \frac{2+1 / n}{n}\right) .
\end{gathered}
$$

Prove that

$$
d\left(\underset{\sim}{t}, \frac{1}{n}\right) \asymp d\left(t, \frac{2+1 / n}{n}\right) .
$$


Obviously, it suffices to prove that

$$
d\left(\underset{\sim}{t}, \frac{1}{n}\right) \succ d\left(\underset{\sim}{t}, \frac{2+1 / n}{n}\right)
$$

Let $t_{1} \in \Gamma_{1+1 / n}, t_{2} \in \Gamma_{1+((2+1 / n) / n)}$ be such that

$$
\begin{aligned}
d\left(\underset{\sim}{t}, \frac{1}{n}\right) & =\left|\underset{\sim}{t}-t_{1}\right|, \quad d\left(\underset{\sim}{t}, \frac{2+1 / n}{n}\right)=\left|\underset{\sim}{t}-t_{2}\right| ; \\
w_{1} & =\varphi\left(t_{1}\right), \quad w_{2}=\varphi\left(t_{2}\right), \quad w=\varphi(t) .
\end{aligned}
$$

Following Belyi [7], we take in the ring

$$
1+\frac{1}{n} \leq|w| \leq 1+\frac{2+1 / n}{n}
$$

a segment and an arc of a circle connecting the points $w_{1}$ and $w_{2}$. Let $l=l\left(w_{1}, \widetilde{w}_{2}\right)$. Construct a family of circles with a center at the point $t$, intersecting $l$. Each of these has an annular arc in $\Omega=C G$, intersecting $l$. We denote a family of such arcs by T. Obviously, the family $\mathrm{T}$ separates in $\Omega$ the point $t_{1}$ and some point $\underset{\sim}{t^{*}}$ (in the simplest cases $\underset{\sim}{t^{*}}=\underset{\sim}{t}$ ) from $t_{2}$ and $\infty$. Therefore, by Lemma A we have

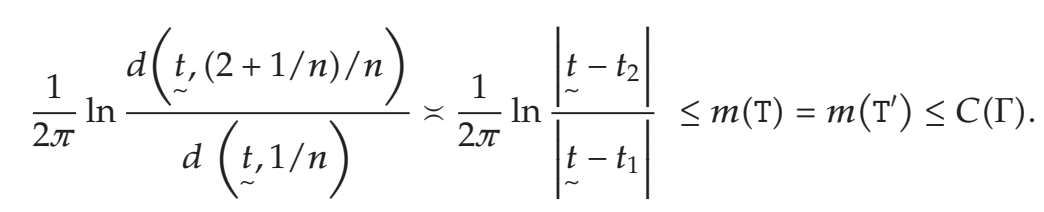

Hence (4.22) and relation (4.21) together with (4.20) prove (4.19),

So, Lemma 4.1 is proved in the case $s \geq 0$.

The proof in the case $s<0$ is conducted by means of analytic reasoning after introducing the auxiliary function

$$
S_{1}(z)=\frac{\left[\varphi^{\prime}(\widetilde{z})\right]^{s} P_{n}(z)}{\varphi^{n+|s|}(z)}
$$

The proof of Lemma 4.2 is conducted in the same way.

Indeed, in the case $s \geq 0$, instead of relation (4.18) from Lemma D we'll have

$$
\left\|\frac{P_{n}(\xi)}{d^{s}(\widetilde{\xi}, \Gamma) \varphi^{n}(\xi)}\right\|_{L_{p}\left(\Gamma_{1+1 / n}\right)} \leq C(P)\left\|\frac{P_{n}(z)}{\tilde{d}^{s}(\tilde{z}, 1 / n) \varphi^{n}(z)}\right\|_{L_{p}(\Gamma)}
$$


Therefore, in order to prove the statement of Lemma 4.2, obviously, it suffices to see the validity of the relation

$$
d(\tilde{\xi}, \Gamma) \asymp d\left(\xi, \Gamma_{R}\right), \quad \xi \in \Gamma_{1+\rho}, \quad \tilde{\xi}=\psi\left(\left(1+\frac{1}{n}\right) \varphi(\xi)\right), \quad R=|\tau|\left(1+\frac{1}{n}\right)
$$

and since the estimation $d\left(\xi, \Gamma_{R}\right) \leq d(\tilde{\xi}, \Gamma)$ is obvious, we have to show that

$$
d(\tilde{\xi}, \Gamma) \preccurlyeq d\left(\xi, \Gamma_{R}\right), \quad \xi \in \Gamma_{1+\rho}, \quad R=|\tau|\left(1+\frac{1}{n}\right), \quad(|\tau|=1+\rho)
$$

This relation is proved exactly in the same way as relation (4.19) in Lemma 4.1.

The case $s \leq 0$ is proved similarly.

Proof of Lemma 4.3. Let $\Gamma \in B_{k}$. Consider two possible cases.

(1) We have $s \leq j$. The case $s=j$ follows from Lemma 4.4.

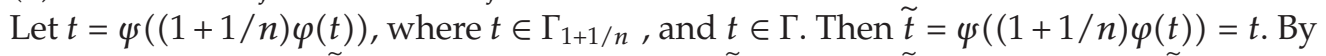
the property of the class $B_{k}$, we will have

$$
d\left(z, \frac{1}{n}\right) \asymp|\tilde{z}-z| \preccurlyeq|\tilde{z}-\underset{\sim}{t}|^{(k-1) / k}|\underset{\sim}{\tilde{t}}-\underset{\sim}{t}|^{1 / k}
$$

and (see [3, page 393])

$$
|t-\underset{\sim}{t}| \asymp \tilde{d}\left(t, \frac{1}{n}\right)
$$

Now, by (4.30) and (4.31), we will get

$$
d\left(z, \frac{1}{n}\right) \preccurlyeq|\tilde{z}-\underset{\sim}{t}|^{(k-1) / k} \tilde{d}^{1 / k}\left(t, \frac{1}{n}\right), \underset{\sim}{\tilde{t}}=t
$$

Hence we will get

$$
B \stackrel{\text { def }}{=} \widetilde{d}^{s}\left(t, \frac{1}{n}\right) \int_{\Gamma} \frac{d^{j-s}(z, 1 / n)|d z|}{|z-t|^{j+1}} \preccurlyeq \widetilde{d}^{s}\left(t, \frac{1}{n}\right) \tilde{d}^{(j-s) / k}\left(t, \frac{1}{n}\right) \int_{\Gamma} \frac{|\tilde{z}-\underset{\sim}{t}|^{((k-1) / k)(j-s)}|d z|}{|z-t|^{j+1}} .
$$

Now, if we take into account $|\tilde{z}-\underset{\sim}{t}| \preccurlyeq|z-t|$ and

$$
|\tilde{z}-\underset{\sim}{t}| \leq|\widetilde{z}-z|+|z-\underset{\sim}{t}| \preccurlyeq d\left(z, \frac{1}{n}\right)+|z-t|+|t-\underset{\sim}{t}| \leq|z-t|+|z-t|+\tilde{d}\left(t, \frac{1}{n}\right) \preccurlyeq|z-t|,
$$


then by Lemma 4.4, for $\Gamma \in S_{\theta}\left(B_{k} \subset S_{\theta}\right)$ we will get

$$
B \preccurlyeq \tilde{d}^{s+(j-s) / k}\left(t, \frac{1}{n}\right) \int_{\Gamma} \frac{|d z|}{|z-t|^{1+s+(j-s) / k}} \preccurlyeq 1 .
$$

(2) We have $j<s<k j /(k-1)$. By the property of the class of curves $B_{k}$, we will have

$$
|t-\underset{\sim}{t}|^{k} \preccurlyeq|t-z|^{k-1}|\tilde{z}-z|,
$$

hence

$$
d\left(z, \frac{1}{n}\right) \succ|\tilde{z}-z| \succ \frac{|t-\underset{\sim}{t}|^{k}}{|t-z|^{k-1}} \succ \frac{\tilde{d}^{k}(t, 1 / n)}{|t-z|^{k-1}} .
$$

Hence, using Lemma 4.4

$$
B \stackrel{\text { def }}{=} \widetilde{d}^{s}\left(t, \frac{1}{n}\right) \int_{\Gamma} \frac{|d z|}{d^{s-j}(z, 1 / n)|z-t|^{j+1}} \preccurlyeq \tilde{d}^{s-k(s-j)}\left(t, \frac{1}{n}\right) \int_{\Gamma} \frac{|d z|}{|z-t|^{1+s-k(s-j)}} \preccurlyeq 1 .
$$

So, Lemma 4.3 is proved.

\section{Proofs of Theorems}

Proof of Theorem 3.3. Consider the case $p=1$. Let $\Gamma$ be an arbitrary rectifiable $K$ quasiconformal curve. By the Cauchy formula, we will have

$$
\begin{aligned}
A \stackrel{\text { def }}{=}\left\|\frac{P_{n}^{(j)}(t)}{\widetilde{d}^{s-j}(t, 1 / n)}\right\|_{L_{1}\left(\Gamma_{1+1 / n}\right)} & =\frac{j !}{2 \pi} \int_{\Gamma_{1+1 / n}} \frac{|d t|}{\widetilde{d}^{s-j}(t, 1 / n)}\left|\int_{\gamma_{t}} \frac{P_{n}(\xi)}{\widetilde{d}^{s}(\xi-t)^{j+1}}\right| \\
& \leq \frac{j !}{2 \pi} \int_{\Gamma_{1+1 / n}} \frac{|d t|}{\widetilde{d}^{s-j}(t, 1 / n)} \int_{\gamma_{t}}\left|P_{n}(\xi)\right||d \xi|,
\end{aligned}
$$

where $\gamma_{t}$ denotes a closed curve containing the point $t$ interior to itself, and that is defined in the following way.

Let the point $t \in \Gamma_{1+1 / n}$ under the mapping $w=\varphi(t)$ go over to the point $u$ (Figure 1 ).

Draw a circle $\gamma_{u}$ with a center at the point $u$ of radius $1 / n$. Denote preimage of this circle under the mapping $z=\psi(w)(w=\varphi(z))$ by $\gamma_{t}$. 


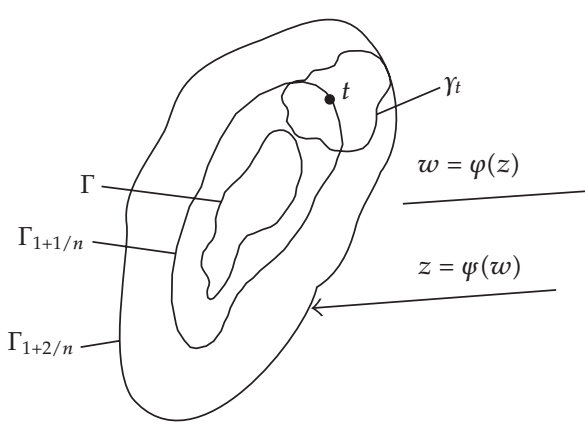

$(z)$

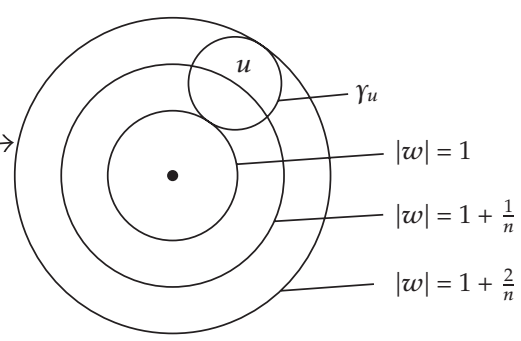

$(w)$

Figure 1 relation

With such a construction of $\gamma_{t}$ it is easy to see that by Lemma C, for all $\xi \in \gamma_{t}$, the

$$
|\xi-t| \asymp \tilde{d}\left(t, \frac{1}{n}\right)
$$

will be valid.

Really, since the relation $|\tau-u|=|u-\underset{\sim}{u}|=1 / n, \underset{\sim}{u}=\varphi(\underset{\sim}{\sim}), \psi(\tau)=\xi, \psi(u)=t$ is valid for all $\tau \in \gamma_{u}$, then by Lemma $C$ we will have

$$
|\xi-t| \asymp|t-\underset{\sim}{t}|
$$

And since

$$
|t-\underset{\sim}{t}| \asymp \tilde{d}\left(t, \frac{1}{n}\right)
$$

(see [7]), then $|\xi-t| \asymp \widetilde{d}(t, 1 / n)$.

Therefore, by Lemma $C$ from relation (5.1) we find

$$
\begin{aligned}
A & \preccurlyeq \int_{\Gamma_{1+1 / n}} \frac{|d t|}{\tilde{d}^{s+1}(t, 1 / n)} \int_{\gamma_{t}}\left|P_{n}(\xi)\right||d \xi| \\
& =\int_{|u|=1+1 / n} \frac{\left|\psi^{\prime}(u)\right||d u|}{\widetilde{d}^{s+1}(\psi(u), 1 / n)} \times \int_{\gamma_{u}}\left|P_{n}(\psi(\tau))\right|\left|\psi^{\prime}(\tau)\right| d \tau|| \\
& \asymp n \int_{|u|=1+1 / n}|d u| \int_{\gamma_{u}} \frac{\left|P_{n}(\psi(\tau))\right|\left|\psi^{\prime}(\tau)\right|}{d^{s}\left(\psi(\tau), \Gamma_{R}\right)}|d \tau|,
\end{aligned}
$$


and under $d\left(\psi(\tau), \Gamma_{R}\right)$ we understand a distance from the point $\xi=\psi(\tau)$ to the level line $\Gamma_{R}$, where $R=|\tau|(1+1 / n)$. Therewith, by Lemma $C$, we take into account that this distance has the same order of $\tilde{d}(t, 1 / n)$, that is,

$$
d\left(\psi(\tau), \Gamma_{R}\right) \asymp \tilde{d}\left(\psi(u), \frac{1}{n}\right) .
$$

Really,

$$
\left|\tau-\tau\left(1+\frac{1}{n}\right)\right|=|\tau-u| \asymp|u-\underset{\sim}{u}| \quad\left(\underset{\sim}{u}=\left(1+\frac{1}{n}\right)^{-1} u\right)
$$

is obvious.

Hence, by Lemma $\mathrm{C}$ it follows that

$$
\left|\psi(\tau)-\psi\left(\tau\left(1+\frac{1}{n}\right)\right)\right|=|\psi(\tau)-\psi(u)| \asymp|\psi(u)-\psi(\underset{\sim}{u})|=|t-\underset{\sim}{t}| .
$$

And since

$$
|t-\underset{\sim}{t}| \asymp \tilde{d}\left(t, \frac{1}{n}\right)=\tilde{d}\left(\psi(u), \frac{1}{n}\right)
$$

(see [7]), then

$$
\left|\psi(\tau)-\psi\left(\tau\left(1+\frac{1}{n}\right)\right)\right| \asymp \tilde{d}\left(\psi(u), \frac{1}{n}\right) .
$$

It remains to show that

$$
d\left(\xi, \Gamma_{R}\right)=d\left(\psi(\tau), \Gamma_{R}\right) \asymp|\bar{\xi}-\xi|=\left|\psi\left(\tau\left(1+\frac{1}{n}\right)\right)-\psi(\tau)\right| .
$$

And since the relation

$$
d\left(\psi(\tau), \Gamma_{R}\right) \leq|\psi(\tau)-\psi(\widetilde{\tau})|
$$

is obvious, it suffices to show that

$$
d\left(\psi(\tau), \Gamma_{R}\right) \succ|\psi(\widetilde{\tau})-\psi(\tau)|
$$




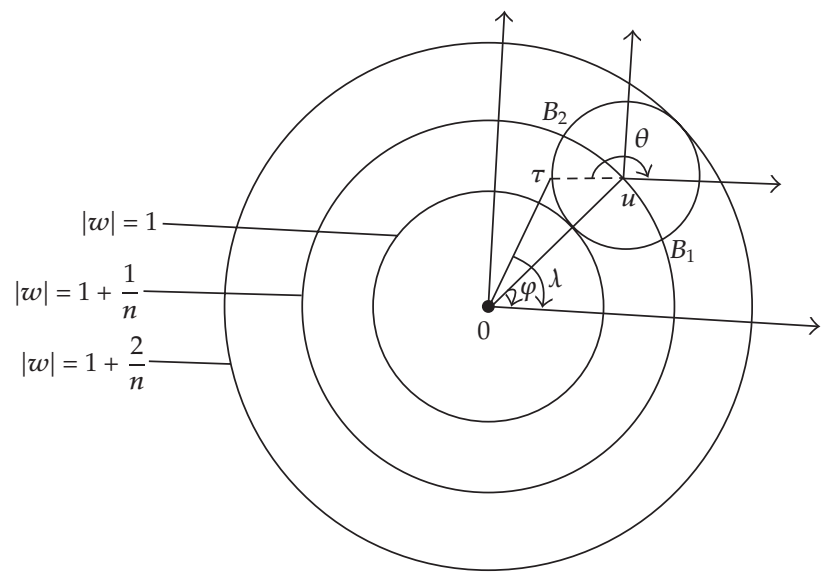

Figure 2

Let $\xi_{0} \in \Gamma_{R}(R=|\tau|(1+1 / n)), \xi_{0}=\psi\left(\tau_{0}\right),\left|\tau_{0}\right|=|\tau|(1+1 / n)$ be a point for which $\left|\xi_{0}-\xi\right|=d\left(\xi, \Gamma_{R}\right)$. Obviously, $\left|\tau_{0}-\tau\right| \geq|\tilde{\tau}-\tau|$. Hence, by Lemma C, it follows the estimation

$$
d\left(\psi(\tau), \Gamma_{R}\right)=d\left(\xi, \Gamma_{R}\right)=|\tilde{\xi}-\xi|=|\psi(\widetilde{\tau})-\psi(\tau)|
$$

that proves relation (5.11) and (5.13); hence the relation (5.6) that we need follows.

Now, in order to estimate the right-hand side of relation (5.5), we divide the circle $\gamma_{u}$ into the arc $\gamma_{1}$, situated interior to the circle $|w|=1+1 / n$ with the ends at the points $B_{1}$ and $B_{2}$ (see Figure 2) and the arc $\gamma_{2}=\gamma_{u} \backslash \gamma_{1}$. In its turn, we divide the arc $\gamma_{1}$ into $\gamma_{1}^{\prime}$ and $\gamma_{1}^{\prime \prime}$, where $\gamma_{1}^{\prime}$, part of the arc $\gamma_{1}$, are situated from the left of the ray ou, connecting the origin of coordinates with the point $u$ and $\gamma_{1}^{\prime \prime}$ from the right of this ray.

Obviously, we will have

$$
A \preccurlyeq n\left(1+\frac{1}{n}\right) \int_{0}^{2 \pi} d \varphi\left\{\int_{\gamma_{1}}+\int_{\gamma_{2}}\right\} \frac{\left|P_{n}(\psi(\tau))\right|\left|\psi^{\prime}(\tau)\right|}{d^{s}(\psi(\tau), 1 / n)}|d \tau|=A_{1}+A_{2} .
$$

Estimate the quantity $A_{1}$ that will be represented in the form

$$
A_{1}=n\left(1+\frac{1}{n}\right) \int_{0}^{2 \pi} d \varphi\left\{\int_{\gamma_{1}^{\prime}}+\int_{\gamma_{1}^{\prime \prime}} \frac{\left|P_{n}(\psi(\tau))\right|\left|\psi^{\prime}(\tau)\right|}{d^{s}(\psi(\tau), 1 / n)}|d \tau|=A_{1}^{\prime}+A_{1}^{\prime \prime} .\right.
$$

Obviously, for the estimation of $A_{1}$, it suffies to estimate the quantity $A_{1}^{\prime}$, since the obtained estimation remains valid for the quantity $A_{1}^{\prime \prime}$ as well, because of symmetric arrangement of $\operatorname{arcs} \gamma_{1}^{\prime}$ and $\gamma_{1}^{\prime \prime}$ with respect to the arc ou.

Let $\tau \in \gamma_{1}^{\prime}$. Then obviously, it will lie on some circle $\gamma_{\rho}$ with center in $o$ and radius equal $1+\rho$, where $\rho \in[0,1 / n]$.

Since $|\tau-u|=1 / n$, then $\tau=u+(1 / n) e^{i \theta}\left(u=\left(1+(1 / n) e^{i \theta}\right)\right)$ (see Figure 2), where $\theta$ is an angle between the ray $\tau u$ and a real axis. Obviously, $\theta=\pi+\varphi-\alpha$, where $\alpha$ is an angle 
between the radii $\tau u$ and $o u$ (see Figure 2) that may be determined by the cosines theorem from the triangle $o \tau u$

$$
\alpha=\arccos \frac{1 / n+\rho^{2} n+2 \rho n+2 \rho}{2(1+1 / n)}=f(\rho)
$$

Hence, we directly have

$$
\tau=u+\frac{1}{n} e^{i(\varphi+\pi-f(\rho))}, \quad d \tau=\frac{i}{n} e^{i(\varphi+\pi-f(\rho))}\left(-f^{\prime}(\rho)\right) d \rho .
$$

Estimating the quantity $A_{1}^{\prime}$, we'll get

$$
\begin{aligned}
& A_{1}^{\prime} \\
& \quad=n\left(1+\frac{1}{n}\right) \int_{0}^{2 \pi} d \varphi \int_{\gamma_{1}^{\prime}} \frac{\left|P_{n}(\psi(\tau))\right|\left|\psi^{\prime}(\tau)\right|}{d^{s}(\psi(\tau), 1 / n)} d t \\
& =\left(1+\frac{1}{n}\right) \int_{0}^{2 \pi} d \varphi \int_{0}^{1 / n} \frac{\left|P_{n}\left(\psi\left(u+(1 / n) e^{i(\pi+\varphi-f(\rho))}\right)\right)\right| \psi^{\prime}\left(u+(1 / n) e^{i(\pi+\varphi-f(\rho))}\right)\left|f^{\prime}(\rho)\right| d \rho}{d^{s}\left(\psi\left(u+(1 / n) e^{i(\pi+\varphi-f(\rho))}\right), 1 / n\right)} \\
& =\left(1+\frac{1}{n}\right) \int_{0}^{1 / n}\left|f^{\prime}(\rho)\right| d \rho \int_{0}^{2 \pi} \frac{\left|P_{n}\left(\psi\left(u+(1 / n) e^{i(\pi+\varphi-f(\rho))}\right)\right)\right|\left|\psi^{\prime}\left(u+(1 / n) e^{i(\pi+\varphi-f(\rho))}\right)\right| d \varphi}{d^{s}\left(\psi\left(u+(1 / n) e^{i(\pi+\varphi-f(\rho))}\right), 1 / n\right)} .
\end{aligned}
$$

Now, making substitution $\tau=(1+1 / \rho-\rho) e^{i \lambda}$ and considering that $\lambda-\varphi=c(\rho)$ (we can determire this from the triangle $о \tau u$ where the sides $o u$ and $\tau u$ are constant by the sines theorem) we will have

$$
\begin{aligned}
A_{1}^{\prime} & =\left(1+\frac{1}{n}\right) \int_{0}^{1 / n} f^{\prime}(\rho) d \rho \int_{0}^{2 \pi} \frac{\left|P_{n}\left(\psi(1+1 / n-\rho) e^{i \lambda}\right)\right|\left|\psi^{\prime}(1+1 / n-\rho) e^{i \lambda}\right| d \lambda}{d^{s}\left(\psi(1+1 / n-\rho) e^{i \lambda}, 1 / n\right)} \\
& =\left(1+\frac{1}{n}\right) \int_{0}^{1 / n} \frac{\left|f^{\prime}(\rho)\right| d \rho}{1+1 / n-\rho} \int_{|\tau|=1+1 / n-\rho} \frac{\left|P_{n}(\psi(\tau))\right||d \tau|}{d^{s}(\psi(\tau), 1 / n)} \\
& \asymp \int_{0}^{1 / n}\left|f^{\prime}(\rho)\right| d \rho \int_{\Gamma_{1+1 / n-\rho}} \frac{\left|P_{n}(\xi)\right|}{d^{s}(\xi, 1 / n)}|d \xi| .
\end{aligned}
$$

Hence, by Lemma 4.2, we will find

$$
A_{1}^{\prime}=C(\Gamma) \int_{0}^{1 / n}\left|f^{\prime}(\rho)\right| d \rho\left\|\frac{P_{n}(z)}{d^{s}(z, 1 / n)}\right\|_{L_{1}(\Gamma)} \leq C(\Gamma)\left\|\frac{P_{n}(z)}{d^{s}(z, 1 / n)}\right\|_{L_{1}(\Gamma)}
$$

As it was said above, this estimation remains valid for the quantity $A_{1}^{\prime \prime}$, as well. 
The same estimation is similarly proved for the quantity $A_{2}$, as well that allows us to see validity of the relation

$$
A \leq C(\Gamma)\left\|\frac{P_{n}(z)}{d^{s}(z, 1 / n)}\right\|_{L_{1}(\Gamma)},
$$

and hence, considering (5.1), the statement of Theorem 3.3 follows for $p=1$ when $\Gamma$ is an arbitrary restifiable $K$-quasiconformal curve. The case $p>1$ is proved similarly. Really, by Lemmas B and C, 4.2, relation (5.6) and relation (5.5), and the Holder inequality, we get

$$
\begin{aligned}
A_{p} & \stackrel{\text { def }}{=} \|\left.\frac{P_{n}^{(j)}(t)}{\tilde{d}^{s}(t, 1 / n)}\right|_{L_{p}\left(\Gamma_{1+1 / n}\right)}=\frac{j !}{2 \pi}\left\{\int_{\Gamma_{1+1 / n}} \frac{|d t|}{\tilde{d}^{(s-j) p}(t, 1 / n)}\left|\int_{\gamma_{t}} \frac{P_{n}(\xi) d \xi}{(\xi-t)^{j+1}}\right|^{p}\right\}^{1 / p} \\
& \preccurlyeq\left\{\int_{\Gamma_{1+1 / n}} \frac{|d t|}{\tilde{d}^{(s+1) p}(t, 1 / n)}\left(\int_{\gamma_{t}}\left|P_{n}(\xi)\right||d \xi|\right)^{p}\right\}^{1 / p} \\
& \asymp\left\{\int_{\Gamma_{1+1 / n}}|d t|\left(\int_{\gamma_{t}} \frac{\left|P_{n}(\xi)\right||d \xi|}{\tilde{d}^{s+1}\left(\xi, \Gamma_{R}\right)}\right)^{p}\right\}^{1 / p} \\
& =\left\{\int_{|u|=1+1 / n}\left|\psi^{\prime}(u)\right||d u|\left(\int_{\gamma_{u}} \frac{\left|P_{n}(\psi(\tau))\right|\left|\psi^{\prime}(\tau)\right||d \tau|}{d^{s+1}\left(\psi(\tau), \Gamma_{R}\right)}\right)^{p}\right\}^{1 / p} \\
& \preccurlyeq\left\{n \int_{|u|=1+1 / n}\left|\psi^{\prime}(u)\right||d u|\left(\int_{\gamma_{u}} \frac{\left|P_{n}(\psi(\tau))\right||d \tau|}{d^{s}\left(\psi(\tau), \Gamma_{R}\right)}\right)^{p}\right\}^{1 / p} \\
& \preccurlyeq n^{1 / p}\left\{\int_{|u|=1+1 / n}\left|\psi^{\prime}(u)\right||d u| \int_{\gamma_{u}}\left|\frac{P_{n}(\psi(\tau))}{d^{s}\left(\psi(\tau), \Gamma_{R}\right)}\right|^{p}|d \tau|\right\}^{1 / p} .
\end{aligned}
$$

Later on, by Lemmas B and C and relation (5.6) it is easy to see the validity of the relation

$$
\left|\psi^{\prime}(u)\right| \asymp\left|\psi^{\prime}(\tau)\right|, \quad|u|=1+\frac{1}{n^{\prime}}, \tau \in \gamma_{u}
$$

where $\gamma_{u}$ is a circle with a center at the point $u$ and of radius equal $1 / 2 n$.

Hence, we directly get

$$
A_{p} \preccurlyeq n^{1 / p}\left\{\int_{|u|=1+1 / n}|d u| \int_{\gamma_{u}} \frac{\left|P_{n}(\psi(\tau))\right|^{p}\left|\psi^{\prime}(\tau)\right|}{d^{s}\left(\psi(\tau), \Gamma_{R}\right)}|d \tau|\right\}^{1 / p} .
$$

Further, the proof is completed in the same way as in the case $p=1$.

So, Theorem 3.3 is proved for the case when $\Gamma \in A_{k}$. The same reasoning allow us to affirm that Theorem 3.3 will be valid in the case $\Gamma \in B_{k}$, as well. 
Finally, we give the proof of Theorem 3.4.

Proof of Theorem 3.4. Let $\Gamma \in B_{k}$ and $s \in[0, k j /(k-1) p)$. Consider the case $p>1$.

Apply the Holder inequality to inner integral of the right-hand side of the relation

$$
\begin{aligned}
A_{p} & \stackrel{\text { def }}{=}\left\|d^{j-s}\left(z, \frac{1}{n}\right) P_{n}^{(j)}(z)\right\|_{L_{p}(\Gamma)} \\
& =\frac{j !}{2 \pi}\left\{\int_{\Gamma} \frac{|d z|}{d^{(s-j)}(z, 1 / n)}\left|\int_{\Gamma_{1+1 / n}} \frac{P_{n}(t) d t}{(t-z)^{(j+1) / p}(t-z)^{(j+1) / q}}\right|^{p}\right\}^{1 / p},
\end{aligned}
$$

where $1 / p+1 / q=1$.

By Lemma 4.5

$$
A_{p} \preccurlyeq\left\{\int_{\Gamma} d^{j-p s}\left(z, \frac{1}{n}\right)|d z| \int_{\Gamma_{1+1 / n}} \frac{\left|P_{n}(t)\right|^{p}|d t|}{|z-t|^{j+1}}\right\}^{1 / p} .
$$

Hence, changing the integration order and applying the statements of Lemmas 4.3 and 4.1 , we get the required inequality (3.10) in the case $p>1$.

In order to see validity of Theorem 3.4 in the case $p=1$, in the right-hand side of the obvious relation

$$
\int_{\Gamma}\left|d^{j-s}\left(z, \frac{1}{n}\right) P_{n}^{(j)}(z)\right||d z| \preccurlyeq \int_{\Gamma} d^{j-s}\left(z, \frac{1}{n}\right) \int_{\Gamma_{1+1 / n}} \frac{\left|P_{n}(t)\right||d t|}{|t-z|^{j+1}}|d z|,
$$

it siffies to change the integration order and apply the statements of Lemmas 4.3 and 4.1.

Remark 5.1. It is easy to show that Theorem 3.4 is valid for any $s \in[0, \infty)$, if $\Gamma$ is fulfiled as the condition (obviously, this condition is always fulfilled if $\Gamma$ is a boundary of an arbitrary convex domain) $\left|\psi^{\prime}(w)\right| \preccurlyeq\left|\psi^{\prime}(1+1 / n) w\right|$ for all $w:|w|=1$.

Really, let $s \geq k j /(k-1) p$. Choose $m>j$ such that the condition $s<k m /(k-1) p$ is fulfilled. Then repeating the reasoning mentioned above in the case $s<k j /(k-1) p$, we get

$$
\left\|\frac{P_{n}^{(m)}(z)}{d^{s-m}(z, 1 / n)}\right\|_{L_{p}(\Gamma)} \leq C(\Gamma, p, m, s)\left\|\frac{P_{n}(z)}{d^{s}(z, 1 / n)}\right\|_{L_{p}(\Gamma)} .
$$

Now, expand the function $P_{n}^{(j)}(z)$ in Taylor's series in the vicinity of the point $\tilde{z}=$ $\tilde{z}(1 / n) \in \Gamma_{1+1 / n}$ :

$$
\begin{aligned}
P_{n}^{(j)}(z)= & P_{n}^{(j)}(\tilde{z})+\frac{P_{n}^{(j+1)}(\tilde{z})(\tilde{z}-z)}{1 !}+\cdots+\frac{P_{n}^{(m-1)}(\tilde{z})}{(m-j-1) !}(\tilde{z}-z)^{m-j-1} \\
& +\frac{1}{(m-j-1) !} \int_{\tilde{z}}^{z}(\xi-z)^{m-j-1} P_{n}^{(m)}(\xi) d \xi
\end{aligned}
$$


Further, divide both parts of this equality into $d^{s-j}(z, 1 / n)$, and consider that $d(z, 1 / n) \succ d\left(\xi, \Gamma_{R}\right)$ (see (5.6)) raise to the $p$ th power, integrate with respect to $\Gamma$ and take the $p$ th power root. We will have

$$
\begin{aligned}
A_{p} \stackrel{\text { def }}{=} & \left(\int_{\Gamma}\left|\frac{P_{n}^{(j)}(z)}{d^{s-j}(z, 1 / n)}\right|^{p}|d z|\right)^{1 / p} \preccurlyeq\left(\int_{\Gamma}\left|\frac{P_{n}^{(j)}(\widetilde{z})}{d^{s-j}(z, 1 / n)}\right|^{p}|d z|\right)^{1 / p} \\
& +\left(\int_{\Gamma}\left|\frac{P_{n}^{(j+1)}(\tilde{z})}{d^{s-(j+1)}(z, 1 / n)}\right|^{p}|d z|\right)^{1 / p}+\cdots+\left(\int_{\Gamma}\left|\frac{P_{n}^{(m-1)}(\tilde{z})}{d^{s-(m-1)}(z, 1 / n)}\right|^{p}|d z|\right)^{1 / p} \\
& +\left(\int_{\Gamma}\left|\int_{z}^{z} \frac{P_{n}^{(m)}(\xi) d \xi}{d^{s-m+1}(\xi, 1 / n)}\right|^{p}|d z|\right)^{1 / p}=A_{p}^{(j)}+\cdots+A_{p}^{(m)} .
\end{aligned}
$$

Now considering Lemmas B and C, 4.1, and Theorem 3.3 and making substitution $\eta=\tilde{z}, z=\psi\left((1+1 / n)^{-1} \varphi(\eta)\right)=\eta$, we get (here in our reasoning we assume, $\left|\psi^{\prime}\left((1+1 / n)^{-1} \varphi(t)\right)\right| \preccurlyeq\left|\psi^{\prime}(\varphi(t))\right|$ for all $\left.\tilde{t} \in \Gamma_{1+1 / n}\right)$ :

$$
\begin{aligned}
A_{p}^{(j)} & \stackrel{\text { def }}{=}\left(\int_{\Gamma}\left|\frac{P_{n}^{(j)}(\tilde{z})}{d^{s-j}(z, 1 / n)}\right|^{p}|d z|\right)^{1 / p} \preccurlyeq\left(\int_{\Gamma_{1+1 / n}}\left|\frac{P_{n}^{(j)}(\eta)}{\widetilde{d}^{s-j}(\eta, 1 / n)}\right|^{p}|d \eta|\right)^{1 / p} \\
& \preccurlyeq\left(\int_{\Gamma}\left|\frac{P_{n}(z)}{d^{s}(z, 1 / n)}\right|^{p}|d z|\right)^{1 / p} .
\end{aligned}
$$

All remaining integrals on the right-hand side of relation (5.31) are similarly estimated except for the last one, for which following the proof of Theorem 3.3 we find

$$
\begin{aligned}
A_{p}^{(m)} & \stackrel{\text { def }}{=}\left(\int_{\Gamma}\left|\int_{\tilde{z}}^{z} \frac{P_{n}^{(m)}(\xi) d \xi}{d^{s-m+1}(\xi, 1 / n)}\right|^{p}|d z|\right)^{1 / p} \\
& \preccurlyeq n^{1 / p}\left(\int_{|w|=1}|d w| \int_{\widetilde{w}}^{w}\left|\frac{P_{n}^{(m)}(\psi(\tau))}{d^{s-m}\left(\psi(\tau), \Gamma_{R}\right)}\right|^{p}\left|\psi^{\prime}(\tau)\right||d \tau|\right)^{1 / p} .
\end{aligned}
$$

Reasoning in the same way as in obtaining estimation (5.5), we'll have

$$
A_{p}^{(m)} \preccurlyeq\left\|\frac{P_{n}(z)}{d^{s}(z, 1 / n)}\right\|_{L_{p}(\Gamma)}
$$

Hence by (5.31) the statement of Theorem 3.4 will follow in the case $s \geq k j /(k-1) p$. So, Theorem 3.4 is proved. 
Remark 5.2. Note that by Lemma 4.4 and the inverse to it of result $1<\gamma \leq 2$ proved in the paper [15], we will have $S_{\theta}=J_{\gamma}(1<\gamma \leq 2)$. Obviously, this result will allow us to derive from Theorems 3.1 and 3.2 the validity of these theorems on arbitrary curves $\Gamma \in S_{\theta}$ as a corollary.

\section{Acknowledgment}

The authors would like to thank the reviewers for carefully checking the manuscript and for his/her valuable comments and suggestions.

\section{References}

[1] S. N. Mergelian, "Some questions of the constructive theory of functions," Proceedings of the Steklov Institute of Mathematics, vol. 37, pp. 3-90, 1951 (Russian).

[2] N. A. Lebedev and P. M. Tamrazov, "Inverse approximation theorems on regular compacta of the complex plane," Mathematics of the USSR-Izvestiya, vol. 34, no. 6, pp. 1340-1390, 1970 (Russian).

[3] V. K. Dzjadyk, Introduction to the Theory of Approximation of Functions by Polynomials, Nauka, Moscow, Russia, 1977.

[4] J. I. Mamedkhanov and I. B. Dadashova, "Singular characteristics in S.N. Bernstein classic estimations," in Modern Problems of Mathematics and Related Questions, pp. 148-152, Scientific Centre of the Russian Academy of Sciences, Dagestan State Tekhnical University, Makhachkala, Russia, 2008.

[5] V. V. Salayev, "Primal and inverse estimations for Cauchy's singular integral on a closed curve," Matematicheskie Zametki, vol. 19, pp. 365-380, 1976 (Russian).

[6] V. V. Andrievskii, "Geometric properties of V. K. Dzyadyk domains," Ukrainian Mathematical Journal, vol. 33, no. 6, pp. 723-727, 1980 (Russian).

[7] V. I. Belyi, "Conformal mappings and approximation of analytic functions in domains with quasiconformal boundary," Matematicheskii Sbornik, vol. 102(144), no. 3, pp. 331-361, 1977 (Russian).

[8] J. I. Mamedhanov, "Estimates of the derivatives of analytic functions on curves," Doklady Akademii Nauk SSSR, vol. 217, no. 3, pp. 526-528, 1974 (Russian).

[9] J. I. Mamedkhanov, "Markov-Bernstein inequalities on V.V.Salayev's curves," in Theory of Functions and Approximations, Proceedings of the Second Saratov Winter School, Saratov, Russia, 1986.

[10] J. I. Mamedkhanov, "Some integral inequalities for polynomials on the curves in complex domain," in Special Questions of Theory of Functions, Baku, Azerbaijani, 1989.

[11] M. I. Andrashko, "Inequalities for a derivative of algebraic polynomial in the metric $L_{p},(p \geq 1)$ in domains with angles," Ukrainian Mathematical Journal, vol. 16, no. 4, pp. 439-444, 1964 (Russian).

[12] V. I. Belyi, Methods of conformal invariants in theory of approximation of functions of a complex variable, Ph.D. thesis, Kiev, Ukraine, 1978.

[13] V. V. Andrievskii, “On pproximation of functions by partial sums of a series in Faber polynomials on continua with a nonzero local geometric characteristic," Ukrainian Mathematical Journal, vol. 32, no. 1, pp. 3-10, 1980 (Russian).

[14] V. V. Andrievskii, "Some properties of continua with a piecewise quasiconformal boundary," Ukrainian Mathematical Journal, vol. 32, no. 4, pp. 435-440, 1980 (Russian).

[15] E. P. Dolzhenko and V. I. Danchenko, "Mapping of sets of finite $\alpha$-measure by means of rational functions," Mathematics of the USSR-Izvestiya, vol. 51, no. 6, pp. 1309-1321, 1987 (Russian). 


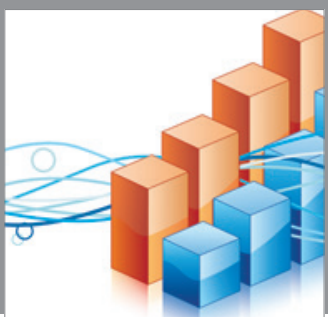

Advances in

Operations Research

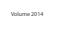

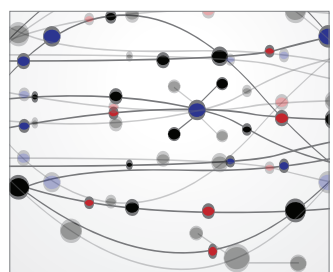

\section{The Scientific} World Journal
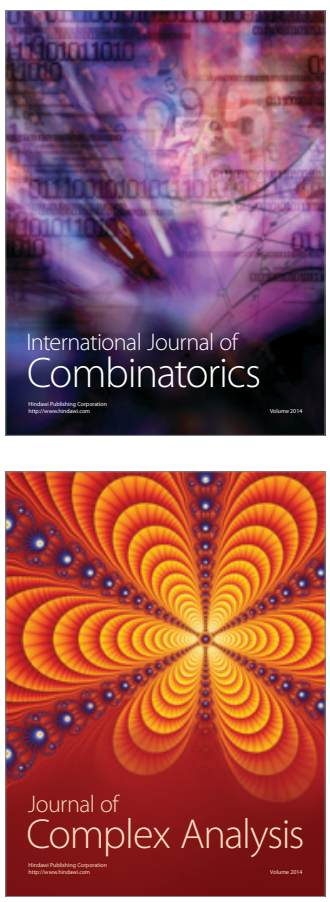

International Journal of

Mathematics and

Mathematical

Sciences
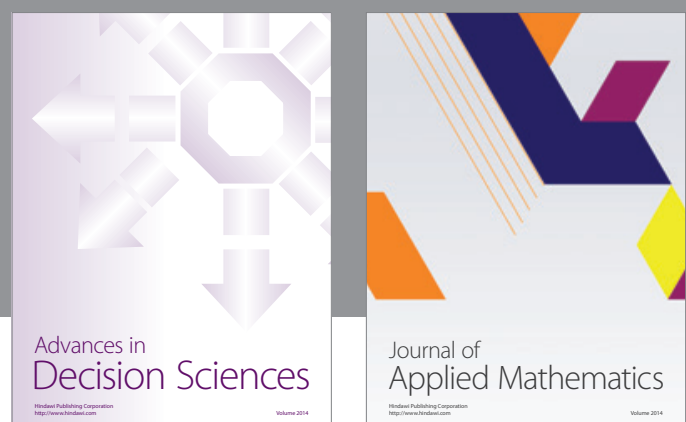

Journal of

Applied Mathematics
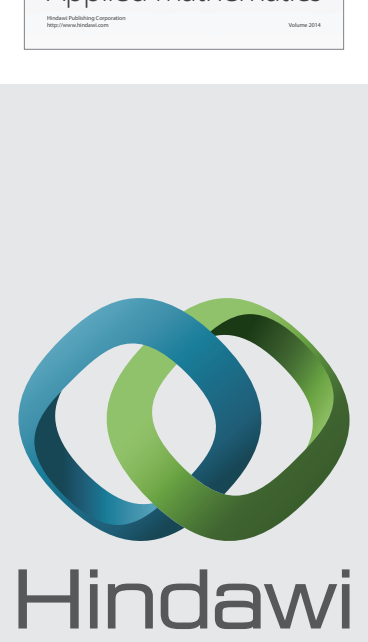

Submit your manuscripts at http://www.hindawi.com
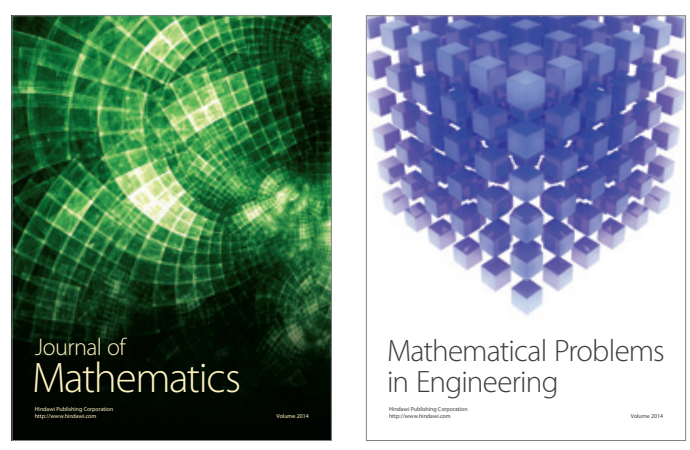

Mathematical Problems in Engineering
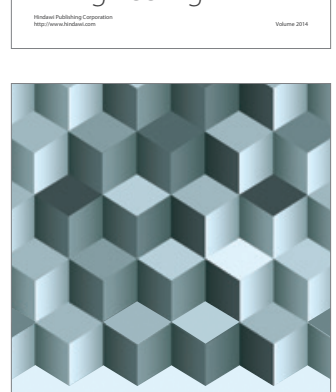

Journal of

Function Spaces
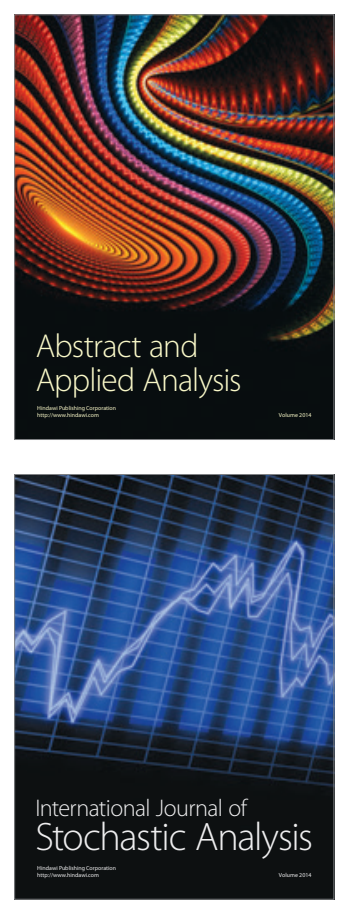

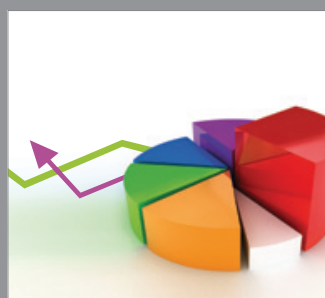

ournal of

Probability and Statistics

Promensencen
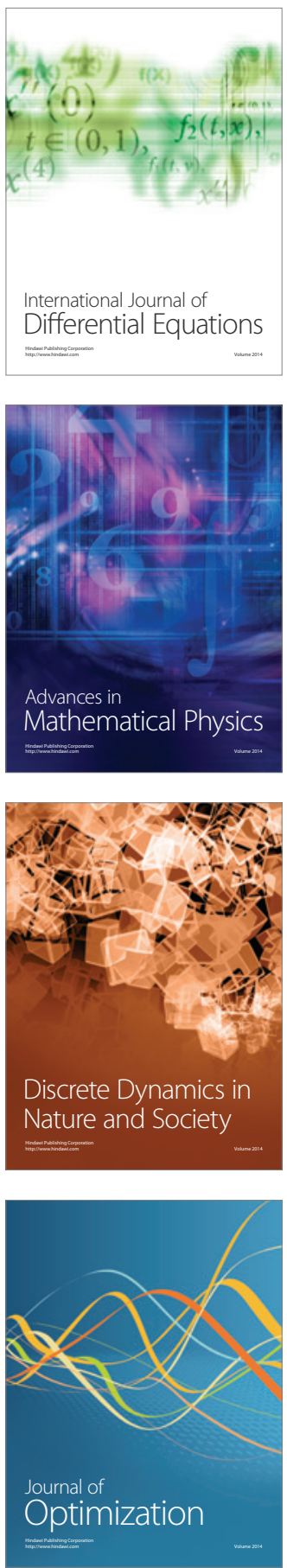